Proceedings of the Edinburgh Mathematical Society (2005) 48, 549-555 (C)

DOI:10.1017/S0013091504000598 Printed in the United Kingdom

\title{
SMYTH SURFACES AND THE DREHRISS
}

\author{
JOHN M. BURNS ${ }^{1}$ AND MICHAEL J. CLANCY ${ }^{2}$ \\ ${ }^{1}$ Department of Mathematics, National University of Ireland Galway, \\ University Road, Galway, Ireland (john.burns@nuigalway.ie) \\ ${ }^{2}$ School of Mathematical Sciences, Dublin City University, \\ Dublin 9, Ireland (michael.clancy@dcu.ie)
}

(Received 7 July 2004)

\begin{abstract}
Isometric deformations of immersed surfaces in Euclidean 3-space are studied by means of the drehriss. When the immersion is of constant mean curvature and the deformation preserves the mean curvature, we determine the drehriss explicitly in terms of the immersion and its Gauss map. These methods are applied to obtain an alternative classification of the Smyth surfaces, i.e. constant mean curvature immersions of the plane into Euclidean 3-space which admit the action of $S^{1}$ as a non-trivial group of internal isometries.
\end{abstract}

Keywords: surfaces; constant mean curvature; isometric deformations; Delaunay

2000 Mathematics subject classification: Primary 3A10

\section{Introduction}

In [3], Smyth posed the following problem. Classify all two-dimensional Riemannian manifolds $(M, g)$ which allow a non-trivial one-parameter group of isometries $\psi_{t}$ : $(M, g) \rightarrow(M, g)$ and for which there exists a constant mean curvature isometric immersion $f: M \rightarrow \mathbb{R}^{3}$. The main result that was obtained in $[\mathbf{3}]$ is now given.

Theorem 1.1 (Smyth). For each integer $m \geqslant 0$ there exists a 1-parameter family of conformal immersions $f_{m}: C \rightarrow \mathbb{R}^{3}$ of constant mean curvature 1 , such that the induced metric is complete and invariant by the group of rotations about 0 . Moreover, 0 is an umbilic of index $-m / 2$, only powers of the rotation through $2 \pi /(m+2)$ about 0 extend to motions of $\mathbb{R}^{3}$ and the associates of $f_{m}$ are given by $\left(f_{m}\right)_{\theta}=f_{m} \circ \mathrm{e}^{-\mathrm{i} \theta}$.

Conversely, any complete surface of constant mean curvature 1 in $\mathbb{R}^{3}$ which admits a one-parameter group of isometries is (to within associates) congruent either to one of the above surfaces $f_{m}$ or to a Delaunay surface.

The purpose of this paper is twofold. Firstly, it is to develop the concept of the drehriss for continuous isometric deformations, especially for immersions $f:(M, g) \rightarrow \mathbb{R}^{3}$, where the deformation preserves mean curvature. Our second purpose is to apply the method of the drehriss to give another characterization of the Smyth surfaces. We note that the 
idea of the drehriss has already found application in the case of infinitesimal deformations (see Liebmann [2] and Blaschke [1] ).

\section{Isometric deformations and the drehriss}

In what follows, $M$ will be an oriented surface with Riemannian metric $g$ and $f^{t}$ : $M \rightarrow \mathbb{R}^{3}$ will be a one-parameter family of orientation-preserving isometric immersions. The positively oriented unit normal field along the immersion $f^{t}$ will be denoted by $\xi^{t}$. Relative to isothermal coordinates $(x, y)$ on a neighbourhood about a fixed point $p \in M$, we write $g=\mathrm{e}^{2 \rho(x, y)}\left(\mathrm{d} x^{2}+\mathrm{d} y^{2}\right)$ and along the curve $t \mapsto f^{t}(p)$ in $\mathbb{R}^{3}$ consider the moving frame $F_{p}^{t}:=\left[f_{x}^{t}(p), f_{y}^{t}(p), \xi^{t}(p)\right]$. For each $t, f^{t}$ is an isometric immersion. Thus, there exists $G_{p}^{t} \in S O(3)$, depending smoothly on $t$, such that $F_{p}^{t}=G_{p}^{t} \cdot F_{p}^{0}$. Furthermore, since $\dot{G}_{p}^{t}\left(G_{p}^{t}\right)^{-1}$ is skew-symmetric, it follows that

$$
\dot{F}_{p}^{t}=\left[\dot{G}_{p}^{t}\left(G_{p}^{t}\right)^{-1}\right] \cdot F_{p}^{t}=\eta^{t}(p) \times F_{p}^{t}
$$

for some uniquely determined $\eta^{t}(p) \in \mathbb{R}^{3}$, where the superposed dot denotes differentiation with respect to $t$ and ' $\times$ ' denotes the cross product in $\mathbb{R}^{3}$. Therefore, for each fixed 'time' $t$ we obtain a map

$$
\eta^{t}: M \rightarrow \mathbb{R}^{3}: p \mapsto \eta^{t}(p),
$$

called the drehriss of the deformation $f^{t}$ at time $t$. When the defining identity $\dot{F}_{p}^{t}=\eta^{t}(p) \times F_{p}^{t}$ is expressed in component form, we obtain

$$
\left.\begin{array}{r}
\dot{f}_{x}^{t}(p)=\eta^{t}(p) \times f_{x}^{t}(p), \\
\dot{f}_{y}^{t}(p)=\eta^{t}(p) \times f_{y}^{t}(p), \\
\dot{\xi}^{t}(p)=\eta^{t}(p) \times \xi^{t}(p) .
\end{array}\right\}
$$

Using the identities $\dot{f}_{x y}=\dot{f}_{y x}$ and $f_{x y}=f_{y x}$, it follows from Equations (2.1) that $\eta_{x} \times f_{y}=\eta_{y} \times f_{x}$ and, consequently,

$$
\left\langle\eta_{y}, \xi\right\rangle \mathrm{e}^{2 \rho}=\left\langle\eta_{y}, f_{x} \times f_{y}\right\rangle=\left\langle\eta_{y} \times f_{x}, f_{y}\right\rangle=\left\langle\eta_{x} \times f_{y}, f_{y}\right\rangle=0 .
$$

With the same argument applied to $\left\langle\eta_{x}, \xi\right\rangle$, we obtain

$$
\left\langle\eta_{x}, \xi\right\rangle=\left\langle\eta_{y}, \xi\right\rangle=0 .
$$

Proposition 2.1. If $A^{t}$ is the Weingarten map corresponding to $f^{t}$ and if $J$ is the positively oriented complex structure on $M$ which is compatible with the metric $g$, then $\eta_{*}=-f_{*} \circ J \circ \dot{A}$.

Proof. Using Equations (2.1), we find from the identity $\xi_{j t}=\xi_{t j}$ that

$$
\xi \times \eta_{j}=f_{*}\left(\dot{A} \frac{\partial}{\partial x^{j}}\right),
$$


and, since $f$ is an isometric immersion preserving the orientation, we have

$$
\xi \times\left(\xi \times \eta_{j}\right)=\xi \times f_{*}\left(\dot{A} \frac{\partial}{\partial x^{j}}\right)=f_{*}\left(J \dot{A} \frac{\partial}{\partial x^{j}}\right) .
$$

Now, using the identity $\xi \times\left(\xi \times \eta_{j}\right)=\left\langle\xi, \eta_{j}\right\rangle \xi-\langle\xi, \xi\rangle \eta_{j}$, together with Equations (2.2), it follows that

$$
\eta_{*} \frac{\partial}{\partial x^{j}}=-\left(f_{*} \circ J \circ \dot{A}\right) \frac{\partial}{\partial x^{j}} \quad \text { for each } j \in\{1,2\},
$$

which proves the proposition.

\section{The determination of $A_{p}^{t}$ and $\eta^{t}$}

We now specialize to the case where the mean curvature is preserved by the deformation. That is, where, for each $p \in M$, the mean curvature $H(p)=\frac{1}{2} \operatorname{Tr} A_{p}^{t}$ is constant in $t$. Recall that, for each $t$, the Weingarten map, $A_{p}^{t}$ is symmetric with respect to $g$ and therefore can be orthogonally diagonalized over the reals. Furthermore, the eigenvalues, $\lambda_{1}(p) \leqslant \lambda_{2}(p)$, of $A_{p}^{t}$ are the roots of the equation $\lambda^{2}-2 H(p) \lambda+K(p)=0$, where $K(p)$ is the Gaussian curvature of $g$ at $p$. In particular, these eigenvalues are independent of $t$ and, therefore, $A_{p}^{t}$ and $A_{p}^{0}$ are orthogonally similar. Thus, when $p$ is not an umbilic (i.e. $\left.\lambda_{1}(p) \neq \lambda_{2}(p)\right)$ there exists a unique $\varphi(p, t) \in[0,2 \pi)$ such that

$$
A_{p}^{t}=\mathrm{e}^{\varphi(p, t) J} A_{p}^{0} \mathrm{e}^{-\varphi(p, t) J} .
$$

It is easy to see that $\left(A^{0}-H I\right)_{p}(-J)=J\left(A^{0}-H I\right)_{p}$, where $I_{p}$ is the identity map on $T_{p} M$. Therefore,

$$
\left(A^{0}-H I\right)_{p} \mathrm{e}^{-\varphi(p, t) J}=\mathrm{e}^{\varphi(p, t) J}\left(A^{0}-H I\right)_{p}
$$

and so we obtain that

$$
\left(A^{t}-H I\right)_{p}=\mathrm{e}^{\varphi(p, t) J}\left(A^{0}-H I\right)_{p} \mathrm{e}^{-\varphi(p, t) J}=\mathrm{e}^{2 \varphi(p, t) J}\left(A^{0}-H I\right)_{p} .
$$

Proposition 3.1. If $f^{t}:(M, g) \rightarrow \mathbb{R}^{3}$ is a one-parameter family of isometric immersions, each having the same constant mean curvature $H$, then

$$
\left(A^{t}-H I\right)_{p}=\mathrm{e}^{\alpha(t) J}\left(A^{0}-H I\right)_{p}
$$

and

$$
\eta^{t}(p)=-\dot{\alpha}(t)\left[\xi^{t}(p)+H f^{t}(p)\right]+\boldsymbol{E}^{t},
$$

where $\boldsymbol{E}^{t} \in \mathbb{R}^{3}$ does not depend on $p$.

Proof. Since both $I$ and $J$ are parallel, it follows from Codazzi's equation that, when $H$ is constant, the angle $\varphi(p, t)$ in Equation (3.1) is independent of $p$. Thus, the first part of the proposition is proved on replacing $2 \varphi(p, t)$ in $(3.1)$ by $\alpha(t)$. To establish the second part, take the $t$-derivative (with $p$ fixed) across the equation 
$\left(A^{t}-H I\right)_{p}=\mathrm{e}^{\alpha(t) J}\left(A^{0}-H I\right)_{p}$ to find $\dot{A}_{p}^{t}=\dot{\alpha}(t) J\left(A^{t}-H I\right)_{p}$. From Proposition 2.1 we have $\eta_{*}=-f_{*} \circ J \dot{A}$. After substituting the latter expression for $\dot{A}$, we find that

$$
\eta_{*}=\dot{\alpha}(t)\left(f_{*} \circ A-H f_{*}\right)=-\{\dot{\alpha}(t)(\xi+H f)\}_{*},
$$

from which the second part of the proposition follows.

Remark 3.2. Since $\left(A^{t}-H I\right)_{p}=\mathrm{e}^{\alpha(t) J}\left(A^{0}-H I\right)_{p}$, the deformation $f^{t}$ is through the associates of $f^{0}$.

\section{The Smyth surfaces}

Here we take $M$ to be $\mathbb{R}^{2}$ with a conformally flat metric $g$ which is invariant under the group of rotations about the origin. Thus, in polar coordinates $(r, \theta)$, we may write $g=\mathrm{e}^{2 \omega(r)}\left(\mathrm{d} r^{2}+r^{2} \mathrm{~d} \theta^{2}\right)$. In this setting, $f^{t}(r, \theta)=f^{0}(r, \theta+t)$, from which it follows that, for all $(r, \theta)$ and $t$, we have

$$
\begin{aligned}
\xi^{t}(r, \theta) & =\xi^{0}(r, \theta+t), \\
A^{t}(r, \theta) & =A^{0}(r, \theta+t) .
\end{aligned}
$$

Combining this observation with the first result in Proposition 3.1, we see that

$$
\left(A^{0}-H I\right)_{(r, \theta+t)}=\left(A^{t}-H I\right)_{(r, \theta)}=\mathrm{e}^{\alpha(t) J}\left(A^{0}-H I\right)_{(r, \theta)} .
$$

In particular,

$$
\mathrm{e}^{\alpha\left(t_{1}+t_{2}\right) J}\left(A^{0}-H I\right)_{(r, \theta)}=\left(A^{0}-H I\right)_{\left(r, \theta+t_{1}+t_{2}\right)}=\mathrm{e}^{\alpha\left(t_{2}\right) J} \mathrm{e}^{\alpha\left(t_{1}\right) J}\left(A^{0}-H I\right)_{(r, \theta)},
$$

so that the function ' $: \mathbb{R} \rightarrow \mathbb{R}: t \mapsto \alpha(t)$ ' is linear and, therefore, $\alpha(t)=c t$ for some constant $c \in \mathbb{R}$. Furthermore, since

$$
\mathrm{e}^{\alpha(2 \pi) J}\left(A^{0}-H I\right)_{(r, \theta)}=\left(A^{0}-H I\right)_{(r, \theta+2 \pi)}=\left(A^{0}-H I\right)_{(r, \theta)},
$$

it follows that the constant $c$ is an integer which we may take to be $-n$ for some integer $n$. Thus $-\dot{\alpha}(t)=n$ and, from the second part of Proposition 3.1, the drehriss of this deformation is given by

$$
\eta^{t}(r, \theta)=n \xi^{t}(r, \theta)+\left(\boldsymbol{E}^{t}+n H f^{t}(r, \theta)\right) .
$$

From the identity $f^{t}(r, \theta)=f^{0}(r, \theta+t)$ it follows that

$$
\begin{aligned}
& \dot{f}^{t}(r, \theta)=f_{\theta}^{0}(r, \theta+t), \\
& \dot{f}_{r}^{t}(r, \theta)=f_{\theta r}^{0}(r, \theta+t), \\
& \dot{f}_{\theta}^{t}(r, \theta)=f_{\theta \theta}^{0}(r, \theta+t), \\
& \dot{\xi}^{t}(r, \theta)=\xi_{\theta}^{0}(r, \theta+t) .
\end{aligned}
$$


On setting $t=0$ and dropping the superscript ' 0 ' from all functions in the previous set of equations, we find (with the help of the moving frame equation $\dot{F}_{p}^{t}=\eta^{t}(p) \times F_{p}^{t}$ ) that

$$
\begin{gathered}
f_{r \theta}(r, \theta)=\dot{f}_{r}(r, \theta)=\eta(r, \theta) \times f_{r}(r, \theta), \\
f_{\theta \theta}(r, \theta)=\dot{f}_{\theta}(r, \theta)=\eta(r, \theta) \times f_{\theta}(r, \theta), \\
\xi_{\theta}(r, \theta)=\dot{\xi}(r, \theta)=\eta(r, \theta) \times \xi(r, \theta) .
\end{gathered}
$$

Now use the formula (4.1) for the drehriss to obtain

$$
\left.\begin{array}{rl}
f_{r \theta} & =n \xi \times f_{r}+(\boldsymbol{E}+n H f) \times f_{r}, \\
f_{\theta \theta} & =n \xi \times f_{\theta}+(\boldsymbol{E}+n H f) \times f_{\theta}, \\
\xi_{\theta} & =(\boldsymbol{E}+n H f) \times \xi .
\end{array}\right\}
$$

However, $\xi \times r f_{r}=f_{\theta}$ and $\xi \times f_{\theta}=-r f_{r}$, so the first two equations above may be written as

$$
\begin{aligned}
& f_{r \theta}=\frac{n}{r} f_{\theta}+(\boldsymbol{E}+n H f) \times f_{r} \\
& f_{\theta \theta}=-n r f_{r}+(\boldsymbol{E}+n H f) \times f_{\theta} .
\end{aligned}
$$

When $n \neq 0$, one obtains from the integrability conditions, $f_{r \theta}=f_{\theta r}$ and $f_{r \theta \theta}=f_{\theta \theta r}$, the additional equation

$$
f_{r r}+\frac{1}{r} f_{r}+\frac{1}{r^{2}} f_{\theta \theta}=\frac{2 H}{r} f_{r} \times f_{\theta} .
$$

This is exactly the condition under which the immersion $f$ has constant mean curvature $H$, because the expression on the left of (4.5) is just the Laplacian of $f$ in polar coordinates. By a change of scale in $\mathbb{R}^{3}$, or on replacing $f$ by $(1 / H) f$, we may assume that $f$ satisfies (4.3)-(4.5) with $H=1$. Furthermore, up to a congruence, we may put $f_{r}(0,0)=\mathrm{e}^{\omega(0)} \boldsymbol{e}_{1}$ and put $f(0, \theta)=\mathbf{0}$ together with $\xi(0, \theta)=\boldsymbol{e}_{3}$, for all $\theta$, where $\boldsymbol{e}_{1}, \boldsymbol{e}_{2}$, $\boldsymbol{e}_{3}$ form the usual basis for $\mathbb{R}^{3}$ and $\omega(0) \in \mathbb{R}$ is the initial value for the metric function $\omega(r)$. With these conventions, we see from the third equation in (4.2) that, when $r=0$, $\mathbf{0}=\xi_{\theta}=\boldsymbol{E} \times \xi$ so that $\boldsymbol{E}=k \xi=k \boldsymbol{e}_{3}$ for some $k \in \mathbb{R}$. Since $f_{r}(0, \theta)$ is obtained by rotating $f_{r}(0,0)$ through $\theta$ about $\boldsymbol{e}_{3}$, it follows that $f_{r \theta}(0, \theta)=\boldsymbol{e}_{3} \times f_{r}(0, \theta)$. Combining this with the first equation in (4.2) when $r=0$, we find that $\boldsymbol{E}=(1-n) \boldsymbol{e}_{3}$. Henceforth, we will assume that the immersion is selected so that the above conditions hold.

Proposition 4.1. With the notation as above, where $f$ is replaced by an appropriate associate, we have

$$
\begin{aligned}
\left\langle(1-n) \boldsymbol{e}_{3}+n f, f_{\theta}\right\rangle & =r^{n} \sin n \theta \\
\left\langle(1-n) \boldsymbol{e}_{3}+n f, f_{r}\right\rangle & =r \mathrm{e}^{2 \omega(r)}-r^{n-1} \cos n \theta \\
\left\langle(1-n) \boldsymbol{e}_{3}+n f, \xi\right\rangle & =r \omega^{\prime}(r)-(n-1) .
\end{aligned}
$$


Proof. If we put $\Psi(r, \theta)=\left\langle(1-n) \boldsymbol{e}_{3}+n f, f_{\theta}\right\rangle$, then it follows from (4.3) that $\Psi_{r}=(n / r) \Psi$ and, therefore, $\Psi(r, \theta)=r^{n} \Gamma(\theta)$ for some smooth function $\theta \mapsto \Gamma(\theta)$. Using (4.3) and (4.4), we find that $\Psi_{\theta \theta}=-n^{2} \Psi$, so $\Gamma(\theta)=\gamma \sin (n \theta+\delta)$ for some constants $\gamma$ and $\delta$. If we replace $f$ by an appropriate associate, we may assume that $\delta=0$, and by making a change of scale in $\mathbb{R}^{2}$ we may assume that $\gamma=1$. This proves the first formula. To establish the second formula, differentiate across the first with respect to $\theta$ and then use Equation (4.4). To prove the third formula, differentiate across the identity $r^{2} \mathrm{e}^{2 \omega(r)}=\left\langle f_{\theta}, f_{\theta}\right\rangle$ with respect to $r$ and then use Equation (4.3).

Remark 4.2. A result similar to that in Proposition 4.1 was obtained in [4], where it was used to show that the immersion $f$ is proper.

Corollary 4.3. If the immersion $f$ is as in Proposition 4.1, then the corresponding Hopf differential is given by $z^{n-2} \mathrm{~d} z^{2}$.

Proof. If we write $\partial_{\zeta}$ to denote $\partial / \partial \zeta$, etc., then

$$
\partial_{z}=\frac{1}{2}\left(\partial_{x}-\mathrm{i} \partial_{y}\right)=\frac{1}{2} \mathrm{e}^{-\mathrm{i} \theta}\left(\partial_{r}-\left(\frac{\mathrm{i}}{r}\right) \partial_{\theta}\right) .
$$

Let $\left(a_{i j}\right)$ denote the matrix representation of the Weingarten map relative to the frame $\left\{\partial_{r},(1 / r) \partial_{\theta}\right\}$. The Hopf differential is given by $\Phi(z) \mathrm{d} z^{2}$, where

$$
\Phi(z)=2 g\left(A \partial_{z}, \partial_{z}\right)=\mathrm{e}^{-\mathrm{i} 2 \theta} \mathrm{e}^{2 \omega(r)}\left\{\left(1-a_{22}\right)-\mathrm{i} a_{21}\right\} .
$$

On the other hand,

$$
-\left(a_{21} f_{r}+\left(\frac{a_{22}}{r}\right) f_{\theta}\right)=-f_{*}\left(A\left(\frac{1}{r}\right) \partial_{\theta}\right)=\xi_{*}\left(\left(\frac{1}{r}\right) \partial_{\theta}\right)=\left(\frac{1}{r}\right) \xi_{\theta} .
$$

Therefore, by the third equation of (4.2) with $\boldsymbol{E}=(1-n) \boldsymbol{e}_{3}$ and $H=1$, we have that

$$
\begin{aligned}
-\left(a_{21} f_{r}+\left(\frac{a_{22}}{r}\right) f_{\theta}\right) & =\left(\frac{1}{r}\right)(\boldsymbol{E}+n f) \times \xi \\
& =\left(\frac{1}{r^{2} \mathrm{e}^{2 \omega(r)}}\right)\left\{(\boldsymbol{E}+n f) \times\left(f_{r} \times f_{\theta}\right)\right\} \\
& =\left(\frac{1}{r^{2} \mathrm{e}^{2 \omega(r)}}\right)\left\{\left\langle\boldsymbol{E}+n f, f_{\theta}\right\rangle f_{r}-\left\langle\boldsymbol{E}+n f, f_{r}\right\rangle f_{\theta}\right\} .
\end{aligned}
$$

It now follows from Proposition 4.1 that $a_{21}=-r^{n-2} \mathrm{e}^{-2 \omega(r)} \sin n \theta$ and that $a_{22}=$ $1-r^{n-2} \mathrm{e}^{-2 \omega(r)} \cos n \theta$. Hence,

$$
\Phi(z)=\mathrm{e}^{-\mathrm{i} 2 \theta} \mathrm{e}^{2 \omega(r)}\left\{\left(1-a_{22}\right)-\mathrm{i} a_{21}\right\}=r^{n-2} \mathrm{e}^{(n-2) \mathrm{i} \theta}=z^{n-2} .
$$

Thus, by replacing $(n-2)$ by $m$ in the above, we have proved the following theorem. 
Theorem 4.4. If, for any integer $m \geqslant 0, f_{m}: C \rightarrow \mathbb{R}^{3}$ is a Smyth surface as described in Theorem 1.1, then, up to a congruence of an associate, $f$ is determined by

$$
\begin{aligned}
& f_{r \theta}=\frac{m+2}{r} f_{\theta}+\left\{(m+2) f-(m+1) \boldsymbol{e}_{3}\right\} \times f_{r}, \\
& f_{\theta \theta}=-(m+2) r f_{r}+\left\{(m+2) f-(m+1) \boldsymbol{e}_{3}\right\} \times f_{\theta}, \\
& f_{r r}=-\frac{1}{r} f_{r}-\frac{1}{r^{2}} f_{\theta \theta}+\frac{2}{r} f_{r} \times f_{\theta},
\end{aligned}
$$

with initial conditions: $f_{r}(0,0)=\mathrm{e}^{\omega(0)} \boldsymbol{e}_{1}$ and $f(0, \theta)=\mathbf{0}$, together with $\xi(0, \theta)=\boldsymbol{e}_{3}$, for all $\theta$, where $\boldsymbol{e}_{1}, \boldsymbol{e}_{2}, \boldsymbol{e}_{3}$ form the usual basis for $\mathbb{R}^{3}$ and $\omega(0)$ is an arbitrary real constant.

\section{References}

1. W. BlaschKe, Vorlesungen über Differentialgeometrie, vol. 1 (Springer, 1930).

2. H. Liebmann, Die Verbiegung von geschlossenen und offenen Flächen positiver Krümmung, Bayer. Akad. Wiss. Math.-Physik Kl. S.-B. (Munich) (1919), pp. 267-291.

3. B. Sмyтн, A generalization of a theorem of Delaunay on constant mean curvature surfaces, in Statistical Thermodynamics and Differential Geometry of Microstructured Materials, Minneapolis, MN, 1991, IMA Volumes in Mathematics and its Applications, vol. 51, pp. 123-130 (Springer, 1993).

4. M. Timmreck, U. Pinkall and D. Ferus, Constant mean curvature planes with inner rotational symmetry in Euclidean 3-space, Math. Z. 215 (1994), 561-568. 